\title{
Article \\ A Novel Energy-Efficient Clustering Algorithm for More Sustainable Wireless Sensor Networks Enabled Smart Cities Applications
}

\author{
Zahid Yousif ${ }^{1}$, Intesab Hussain ${ }^{1}$, Soufiene Djahel ${ }^{2, *}$ (I) and Yassine Hadjadj-Aoul ${ }^{3}$ \\ 1 Department of Telecommunication Engineering, QUEST, Nawabshah 67450, Sindh, Pakistan; \\ engr.zahid.yousif@gmail.com (Z.Y.); intesab@quest.edu.pk (I.H.) \\ 2 Department of Computing and Mathematics, Manchester Metropolitan University, All Saints, \\ Manchester M15 6BH, UK \\ 3 Department of Computer Science, University of Rennes, Inria, CNRS, IRISA, 35000 Rennes, France; \\ yassine.hadjadj-aoul@irisa.fr \\ * Correspondence: s.djahel@mmu.ac.uk
}

check for

updates

Citation: Yousif, Z;; Hussain, I.; Djahel, S.; Hadjadj-Aoul, Y. A Novel Energy-Efficient Clustering

Algorithm for More Sustainable Wireless Sensor Networks Enabled Smart Cities Applications. J. Sens. Actuator Netw. 2021, 10, 50. https:// doi.org/10.3390/jsan10030050

Academic Editors: Giovanni Pau and Fabio Arena

Received: 9 April 2021

Accepted: 25 June 2021

Published: 19 July 2021

Publisher's Note: MDPI stays neutral with regard to jurisdictional claims in published maps and institutional affiliations.

Copyright: (c) 2021 by the authors. Licensee MDPI, Basel, Switzerland. This article is an open access article distributed under the terms and conditions of the Creative Commons Attribution (CC BY) license (https:// creativecommons.org/licenses/by/ $4.0 /)$.

\begin{abstract}
Wireless Sensor Networks (WSNs) is a major sensing technology that has revolutionized the way information is collected, processed, and used in many smart cities' applications that rely on sensing technologies for event detection and monitoring. Despite the multiple benefits that such technology offers, the quick depletion of sensors' battery power represents a major concern, mainly due to the extensive computational tasks and communication operations performed by individual sensors. Indeed, the cost of replacing batteries can be prohibitively expensive, especially when sensors are deployed in areas where access is difficult, in urbanized cities. To extend sensors' lifetime, this paper proposes a new variant of LEACH protocol named LEACH enhanced with probabilistic cluster head selection (LEACH-PRO). LEACH-PRO introduces several measures to extend WSNs nodes' lifetime such as cluster head node selection using a probabilistic function based on maximum residual energy and minimum distance to the sink. The obtained simulation results have proven the supremacy of LEACH-PRO over LEACH and direct transmission protocol in terms of the achieved network lifetime and the generated traffic overhead. Most importantly, LEACH-PRO will significantly extend the sensors' lifetime, which would make this type of deployment more viable in smart city scenarios.
\end{abstract}

Keywords: wireless sensor networks; energy-efficiency; smart cities; LEACH

\section{Introduction}

The concept of smart cities continues to attract a lot of interest from industry, academia and policy makers to enable better quality of life for cities' inhabitants. At the forefront of these cities, sensors and actuators are the main driver for many value-added services and the expected automation, which not only targets the improvement of the urban life, but also the reduction of the management costs of these cities [1]. In order to support the provision of these new services, Wireless Sensor Networks (WSNs) and other similar sensing technologies should be deployed all over cities [2]. Once deployed, these different sensors automatically organize themselves, forming an ad hoc network, in order to ensure global connectivity, regardless of their ranges, which are sometimes very limited. Once deployed and configured, such sensors allow the monitoring of multiple metrics such as motion, temperature or humidity level; they could be responsible for generating certain events, such as the detection of a fire, to take safety measures in real-time [3]; they could even trigger actions, directly or indirectly, such as turning on the light when detecting movements.

WSNs are composed of tiny, low powered, low cost and light weighted sensor nodes. These nodes are capable of sensing, processing, aggregating and transmitting data on the wireless channel directly to the base station (BS) or via neighbor nodes to BS [4]. Usually, 
WSNs are deployed in remote sensing fields where each node is equipped with a nonreplaceable battery and consumes an amount of energy while sensing, aggregating and transmitting data [4]. Hence, communication among sensor nodes must be efficient for faster relief operations in emergencies. In some situations, like areas where accessibility is difficult, it is almost impossible or too costly to replace the exhausted batteries [5]. Since the lifetime of a sensor node depends on its battery or power source capacity, which is consumed through communication and sensing activities, it is necessary to design energy-aware solutions to increase the lifetime of WSNs [6]. The design of energy-efficient routing techniques for WSNs is very challenging task due to the limited computational and storage capacities in such tiny and energy-constrained sensors $[7,8]$. In this paper, we propose a new protocol called LEACH-PRO (LEACH enhanced with probabilistic cluster head selection), which represents an enhanced version of LEACH (low energy adaptive clustering hierarchy), in order to maximize WSNs' lifetime.

The main contribution of this paper is to overcome LEACH's related issues, outlined in Section 5, by designing a new probabilistic mechanism in which $\mathrm{CH}$ selection is made based on two metrics: The maximum residual energy and the minimum distance to the base station. While LEACH-PRO has similar design logic as LEACH, the main difference lies in the $\mathrm{CH}$ selection process and the fact that sensors located at the border of the sensing field are excluded from participating in the $\mathrm{CH}$ selection process under certain conditions, making LEACH-PRO more energy efficient than LEACH. An extensive performance evaluation is performed under varying network densities by measuring several metrics of interest and the results have proved the effectiveness of LEACH-PRO. Moreover, one of the distinguishing features of LEACH-PRO is its compatibility with other state of the art approaches, such as those concerned with the determination of the number of clusters and could therefore be used jointly to complement them.

The remainder of this paper is organized as follows. In Section 2, we provide a brief overview of the importance of wireless sensor networks in smart cities. In Section 3, we present an overview of LEACH routing protocol followed by the literature review in Section 4. Afterwards, we introduce our proposed scheme, named LEACH-PRO, and discuss its operation in detail in Section 5. In Section 6, we present and analyze the obtained simulation results. Finally, we conclude the paper in Section 7.

\section{WSNs Enabled Smart Cities Applications}

To deal with the expanding demography of urban centers, the development of smart cities is becoming more and more critical. These cities will indeed improve the quality of life of citizens by optimizing their daily lives (e.g., urban transportation planning), their safety (e.g., rapid response in emergency situations), while offering them value-added services (e.g., remote monitoring and control of their homes). On the other hand, these cities are expected to integrate intelligence, in order to automate their operation, and should foster energetic sobriety, for a more sustainable and resilient world [9].

The deployment of heterogeneous wireless network technologies will be necessary to enable these advances. Long-range wireless networks, such as 5G or Beyond 5G (B5G) networks, will definitely be needed to serve bandwidth-intensive or low-latency applications. These cities will also require sensor networks, whether short-range, such as WSNs, or long-range, such as Low Power Wide Area Networks (LPWANs), which could serve as a base station (or sink) to aggregate and report sensors readings.

Without being exhaustive, WSNs can be considered in several use cases in smart cities. Their use within smart road infrastructure and integration with Connected Autonomous Vehicles (CAVs) will help preventing accidents while improving traffic flow fluidity. Their use in buildings can reduce energy consumption when combined with actuators. They can trigger alerts, faster, during fires, and allow to adapt, for example, the frequency of the means of transportation according to the needs, enabling a significant reduction in carbon footprint. The number of applications and practical use cases are extremely numerous and are only limited by the boundaries of the human imagination [10]. 
By organizing themselves in networks, WSNs potentially cover large areas, even inside buildings [11]. However, the viability of these sensors is very much linked to their lifetime which should be of several years. To maximize the operating lifetime of these wireless sensors, several strategies have been proposed in the literature. These techniques can be broadly divided into three categories: (i) Strategies based on energy harvesting from the environment (stemming from different sources such as solar, mechanical, or electromagnetic energy) [12], (ii) strategies optimizing the idle time of the devices [13], and (iii) techniques aiming to optimize the wireless devices' operation [14]. These different strategies can be found in different scenarios for smart cities. They are in no way contradictory, and can even be leveraged by the same wireless sensors.

In this paper, we focus on the operational optimization of sensors, and particularly on clustering techniques. Thus, our contribution may concern the various introduced use cases, in which clustering strategies are central for enhanced energy savings. Please note that all notations used in the rest of the manuscript are summarized and explained in Table 1.

Table 1. Summary of the used notations.

\begin{tabular}{|c|c|}
\hline Notation & Meaning \\
\hline $\mathrm{CH}$ & Cluster Head \\
\hline $\mathrm{CM}$ & Cluster Member \\
\hline BS & Base Station \\
\hline$l$ & Length of Message in Bits \\
\hline$p$ & Probability to Select a Node as $\mathrm{CH}$ \\
\hline$N_{i}$ & $i_{\text {th }}$ Node \\
\hline$T\left(N_{i}\right)$ & Threshold Function Value against $i_{t h}$ Node \\
\hline$R\left(N_{i}\right)$ & Random Number against $i_{t h}$ Node \\
\hline DT & Direct Transmission \\
\hline $\mathrm{AP}$ & Access Point \\
\hline G & Set of Nodes not selected as $\mathrm{CH}$ \\
\hline RSS & Received Signal Strength \\
\hline TDMA & Time Division Multiple Access \\
\hline$r$ & Number of Rounds \\
\hline$d$ & Remote Distance to BS \\
\hline$E_{b} / N_{0}$ & Bit to Noise Ratio \\
\hline$E_{A V G}$ & Average Energy of System \\
\hline$E_{C H}$ & Current Energy of $\mathrm{CH}$ \\
\hline$E_{C M}$ & Current Energy of $\mathrm{CM}$ \\
\hline$D_{C M}$ & Distance of CM to BS \\
\hline$D_{A V G}$ & Average Distance to BS \\
\hline$E_{T x-e l e c}$ & Transmitter Electronics \\
\hline$E_{R x-e l e c}$ & Receiver Electronics \\
\hline$\epsilon_{a m p}$ & Transmitter Amplifier \\
\hline ADVPKT & Advertisement Message Packet \\
\hline CTRPKT & Control Packet \\
\hline DRN & Death Rate of Nodes \\
\hline FND & First Node Died \\
\hline HND & Half of the Nodes Died \\
\hline SRE & System Remaining Energy \\
\hline$C_{i d}$ & Cluster ID \\
\hline
\end{tabular}

\section{Background and Overview of LEACH}

In WSNs, recharging the batteries of sensor nodes is an infeasible task [3]. Hence, the network lifetime is a primary concern in WSNs. In order to prolong the network lifetime, several routing protocols exist and could be classified into two types depending on the network topology: Flat routing protocols and Hierarchical routing protocols. Since flat routing protocols require maintaining routing table data and cannot aggregate the sensed data, they are not applicable for large scale WSNs. However, hierarchical routing 
protocols can solve this issue to some extent. Direct Transmission (DT) [4] is the basic hierarchical routing protocol used for transferring data from source node to the destination directly without any intermediate node. Due to this, DT consumes more power for sending information towards the BS, especially when this latter is located far away from the sensing field. This will quickly degrade nodes' battery and reduce the overall lifetime of the network [4].

\subsection{First Order Radio Model}

Low energy radio model is considered as an important factor to communicate effectively over a network. In our work, simple energy consumption first order radio model is presumed. However, energy consumption in transmitter and receiver modes will affect and compensate the performance of routing protocols. In transmitter and receiver mode electronic circuitry dissipate $E_{T x-\text { elec }}=E_{R x \text {-elec }}=E_{\text {elec }}=50 \mathrm{~nJ} /$ bit to run different operations of a given radio model. At the transmitter end the signal is amplified to $\epsilon=100 \mathrm{pJ} / \mathrm{bit} / \mathrm{m}^{2}$ for achieving acceptable bit to noise ratio $E_{b} / N_{0}$ at the receiver end, as shown in Table 2 . Generally, this model describes the energy consumption while transmitting and receiving a packet to neighbor node or to an end station. Equations (1) and (2) show the mathematical model for energy dissipation when transmitting a $(l)$ bit packet to remote distance $(d)$.

$$
\begin{gathered}
E_{T x}(l, d)=E_{T x-e l e c}(l)+E_{T x-e m p}(l, d) \\
E_{T x}(l, d)=E_{\text {elec }} \times l+\epsilon_{\text {amp }} \times l \times d^{2}
\end{gathered}
$$

Equations (3) and (4) describe the energy dissipation induced by the reception of $(l)$ bits packet. In this model, the receiver consumes less energy than the transmitter [15] because it does not need to further amplify the signal for forwarding the received information, but instead will only process it.

$$
\begin{gathered}
E_{R x}(l)=E_{R x-\text { elec }}(l) \\
E_{R x}(l)=E_{\text {elec }} \times(l)
\end{gathered}
$$

Symmetric radio channel is assumed in this model, in which the energy required to transfer information from a node $X$ to a node $Y$ is similar to that needed to transmit the same information from node $Y$ to node $X$. Alternatively, the clustering protocols are designed in such a way that consumes minimum amount of energy for each transition. Additionally, transmit distance should also be reduced for improving the energy consumption for transmission and receiving operation, especially in this model [4,16-18].

Table 2. Radio characteristics.

\begin{tabular}{cc}
\hline Operation & Energy Dissipaton \\
\hline $\begin{array}{c}\text { Transmitter Electronics }|| E_{T x-\text { elec }} \\
\text { Receiver Electronics }|| E_{R x-\text { elec }} \\
\left(E_{T x-\text { elec }}=E_{R x-\text { elec }}=E_{\text {elec }}\right)\end{array}$ & $50 \mathrm{~nJ} / \mathrm{bit}$ \\
\hline Transmitter Amplifier $\left(\in_{\text {amp }}\right)$ & $100 \mathrm{pJ} / \mathrm{bit} / \mathrm{m}^{2}$ \\
\hline
\end{tabular}

\subsection{Direct Transmission (DT)}

DT is the basic routing protocol used in WSNs to route the data from the sensing field directly, without any intermediate node or AP (Access Point), towards the base station. The deployed nodes in the field transmit the sensed data directly to the BS without any intermediate or neighbor node intervention. The nodes located far away from the BS will consume more energy, as opposed to the closer ones, because the energy consumption is directly proportional to the distance separating the sender node from 
the BS. Such transmission operation will rapidly deplete the nodes' battery, which in turn leads to degradation of overall network lifetime. The BS is considered as a remote monitoring station equipped with sufficient power for processing the received data from the sensing filed.

To better understand the energy consumption in DT, consider a linear simple network model composed of $n$ sensor nodes separated by uniformly distributed distance $r$. Equations (5)-(7) describe the energy consumption needed for transmitting $l$ bits long message from the sensing field to the BS in this network. For communication and data transmission, DT uses first order radio model explained in Section $3.1[4,18]$.

$$
\begin{gathered}
E_{\text {direct }}=E_{\text {Tx-elec }}(l, d=n \times r) \\
E_{\text {direct }}=E_{\text {elec }} \times l+\epsilon_{\text {amp }} \times l \times(n \times r)^{2} \\
E_{\text {direct }}=l \times\left(E_{\text {elec }}+\epsilon_{\text {amp }} n^{2} r^{2}\right)
\end{gathered}
$$

\subsection{LEACH Overview}

Due to their tiny size, WSNs' nodes have limited energy resources, meaning that energy-efficient routing is compulsory to maximize the network lifetime. As discussed in Section 2, several energy-efficient routing protocols have been proposed for WSNs in the literature, each of them has its advantages and limitations. However, hierarchical and cluster based routing techniques are the most prominent ones as they foster more energy efficient operations than other alternative schemes. Low Energy Adaptive Clustering Hierarchy $(\mathrm{LEACH})$ is considered as one of the most important basic cluster-based routing protocols used for efficient communication in WSNs. A clustering algorithm ensures optimal energy utilization as compared with non-clustering routing algorithms [4]. LEACH is a basic energy efficient routing protocol that minimizes the power consumption in all aspects of communication, thus improving the network lifetime. In LEACH, the network is divided into a number of clusters, each cluster comprises a cluster head $(\mathrm{CH})$ and a set of cluster members (CMs) as shown in Figure 1. CMs sense and aggregate data from the sensing field and transfer it to the $\mathrm{CH}$. $\mathrm{CH}$ are then responsible for transmitting this data to the BS. LEACH uses randomized selection of $\mathrm{CH}$ to give equal chance to all participating nodes for competing in $\mathrm{CHs}$ selection process rather than using a pre-determined selection of CHs. In static clustering, CHs usually transmit data continuously, consume more power, and die quickly. LEACH operates in rounds; each round consists in two phases, setup phase and steady state phase. In setup phase, a $\mathrm{CH}$ is selected based on the generated random number $R\left(N_{i}\right)$ between zero and one. If the random number $R\left(N_{i}\right)$ is lower than the threshold function $T\left(N_{i}\right)$, a $\mathrm{CH}$ node is elected as shown in Equation (8) [4].

$$
T\left(N_{i}\right)= \begin{cases}\frac{p}{1-p \times\left(\operatorname{rmod} \frac{1}{p}\right)} & \text { if } N_{i} \in G \\ 0 & \text { Otherwise }\end{cases}
$$

where $p$ denotes the probability of selecting a node as $\mathrm{CH}$ in $r$ rounds, and $\mathrm{G}$ represents a set of nodes that have not been selected as $\mathrm{CHs}$ in $1 / p$ rounds. The nodes selected as $\mathrm{CHs}$ generate an advertisement message and broadcast it to $\mathrm{CMs}$; the CMs will then join one of the clusters depending on the maximum received signal strength (RSS). The $\mathrm{CH}$ creates a Time Division Multiple Access (TDMA) slot for each CM for collision free intra cluster communication. In steady state phase, $\mathrm{CH}$ compress, aggregate and transmit data to the BS. In LEACH, the number of transmissions is reduced by introducing the clustering. Efficient CHs selection reduces the power consumption of individual nodes, which maximizes the overall network lifetime [3]. 




Figure 1. Example of a WSN composed of a number of clusters.

\subsubsection{Setup Phase}

LEACH completes its operation in rounds, each round starts with a setup phase followed by a steady state phase. Initially, a cluster organizes itself in the setup phase and completes its advertisement operation. Then, in the steady state phase the CMs transmit data to the $\mathrm{CH}$ and this latter transfers the aggregated data to the BS $[4,18]$.

When the clusters are created, each node individually decides whether to participate in $\mathrm{CH}$ selection operation in the current round or not. Equation (8) shows the formula for calculating $T\left(N_{i}\right)$ threshold value where $N_{i}$ is a node and $p$ is the probability of node $N_{i}$ to become the $\mathrm{CH}$ in round $r$. $\mathrm{G}$ is a set of nodes that are not selected as $\mathrm{CH}$ in previous $1 / p$ rounds. Based on these parameters the threshold value is calculated for further setup operations [7].

Regardless of the network density, the decision is made up on the initial probability (i.e., the probability $p$ used in Equation (8) of the nodes that will become the $\mathrm{CH}$. Afterwards, those nodes get a chance to become the $\mathrm{CH}$ for the current $1 / p$ rounds. $\mathrm{CHs}$ broadcast an advertisement (ADV) message to all nodes. Upon receiving an ADV message, CMs send a response message to their chosen $\mathrm{CH}$ individually based on the measured RSSI. Each $\mathrm{CH}$ creates a TDMA schedule and sends it to its CMs to avoid the collision while transmitting data to $\mathrm{CH}$. Each $\mathrm{CM}$ receives the TDMA schedule and accordingly switches to sleep state until its scheduled time slot is reached. At this instant only, this CM can send the data. The operation of TDMA schedule creation is illustrated in Figure 2, where $T S_{i}$ refers to Time Slot $i$. 




Figure 2. LEACH frame operation.

\subsubsection{Steady State Phase}

At the end of the setup phase operation, every $\mathrm{CM}$ can start the data transmission towards its $\mathrm{CH}$ during its own assigned time slot. We assume that each $\mathrm{CM}$ has always data to transmit. Regardless of the communication in each cluster, the $\mathrm{CH}$ must keep its radio receiver on for receiving all data from its CMs. In addition, when TDMA time slot is over or a round time has ended the $\mathrm{CH}$ performs data compression over the received data from its CMs and sends the resulting data to the BS. As the distance from the BS to the $\mathrm{CH}$ is significantly larger than the distance separating this latter and its $\mathrm{CMs}$, this transmission requires high-energy consumption compared to other data transmission operations. This whole process represents the steady state phase operation of LEACH. Subsequently, the sensor nodes which have not been elected as $\mathrm{CH}$ in previous rounds will participate to become $\mathrm{CH}$ in next rounds.

\section{Related Work}

In this section, we will provide a brief overview of the most important works that have proposed extensions or improvements to the basic LEACH protocol discussed in the previous section.

In [19], the authors proposed an effective differential evolution (DE) based routing algorithm, named DE-LEACH, to improve the performance of LEACH. DE-LEACH's CHs selection process is similar to LEACH, i.e., random selection based on threshold value shown in Equation (8). DE-LEACH is designed by leveraging two of the most important factors of WSNs, which are energy consumption and the distance separating neighboring nodes inside a cluster, to overcome the early death of $\mathrm{CH}$ issue. DE-LEACH features a fast search mechanism based on optimum level while selecting $\mathrm{CH}$ in the setup phase. Performance evaluation results, using simulation, show that DE-LEACH can successfully prevent random selection of nodes during the clustering process while improving largescale WSNs lifetime. However, the exclusion of random selection of $\mathrm{CHs}$ using optimal level based on residual energy increases the complexity of the algorithm and the overhead in the network [19].

In Energy-Efficient LEACH (EE-LEACH) protocol, proposed in [20], the $\mathrm{CH}$ selection is based on the residual energy where a sorting algorithm extracts the residual energy of each sensor node and the one which has the maximum residual will be chosen as a $\mathrm{CH}$. Gaussian distribution is used for computing the coverage probability. The collection of data can save substantial energy despite the fact that one cluster formed in a small area can consume less energy than the cluster formed in a larger one. The experimental evaluation of EE-LEACH highlights that it has higher packet delivery ratio, lower end-to-end delay and lower energy consumption, but It achieves this at the cost of more traffic on the network. In Intra Cluster Balanced Cluster LEACH (IB-LEACH Protocol) [21], the clustering process is divided into two phases, i.e., intra cluster and inter cluster, which will further help to increase the energy efficiency of the system. The evaluation results reveal that the use of 
IB-LEACH protocol increases the network lifetime. On the other hand, due to the extra computing required it increases the traffic load to some extent.

Assisted LEACH (A-LEACH) [22] is an amplified form of LEACH aiming to minimize the power dissipation of $\mathrm{CHs}$ by introducing a helper node, called assistant node, to the cluster head. In A-LEACH, when a cluster is formed and the $\mathrm{CH}$ is selected this latter selects a helper node located near the base station. Then, the cluster head aggregates the received data from the cluster members and sends it, through the selected helper node, to the BS. The evaluation results has shown that A-LEACH performs better than LEACH.

Balanced-LEACH (LEACH-B) uses de-centralized approach for cluster formation in which each node knows the final destination to transmit data to and its own position but has no information about other nodes [23]. LEACH-B operation consists of three steps, cluster formation, $\mathrm{CH}$ selection and data transmission. A sensor node sends data to destination node using the best path characterized by the low power dissipation and based on that it selects the $\mathrm{CH}$. Firstly, the great convergence and global optimization capability of the proposed algorithm can reasonably divide the entire network area into a number of sub-regions. Then, in each sub-region a $\mathrm{CH}$ is elected taking the residual energy factor into account. The conducted experiments results have shown that, compared to LEACH, LEACH-B can effectively balance the network load and improve the energy usage, so as to extend the network lifetime. However, the division of the entire network into sub-regions increases the communication overhead in WSNs [23].

In Round Robin Cluster Head (RRCH) [24], clusters are created one time only, using similar method to LEACH, in order to minimize the energy usage during the clustering phase. Once the clusters are formed, $\mathrm{RRCH}$ keeps the clusters fixed and uses the round-robin scheme to select the $\mathrm{CH}$ within each cluster. All nodes have equal chance to be $\mathrm{CH}$ during each frame. Upon detection of an abnormal node, the $\mathrm{CH}$ modifies the scheduling information and broadcasts it to the entire cluster during the frame modification. Afterwards, its cluster members delete this abnormal node based on the received updated schedule information. Similar to LEACH, RRCH suffers from the incapability of guaranteeing a good quality of the formed clusters. Without the periodic re-clustering (i.e., regeneration of clusters), RRCH cannot manage clusters with varying sizes (e.g., overlay of clusters, very small or very large clusters).

Fixed LEACH (LEACH-F) [25] is centralized technique of cluster formation in which the clusters are formed permanently by the base station, only the CHs are changed within a cluster in rotation. In default LEACH, re-clustering is performed in each round, whereas, LEACH-F removes re-clustering process in next rounds for the whole network lifetime. The steady state is similar to LEACH. LEACH-F is not flexible due to preemptive policy of clusters formation. Before the completion, in each round, energy and information are wasted due to fixed round time in the steady state. Because the CHs or CMs in each cluster cannot change their behavior, this results in an uncertainty in the whole network and degrades the overall performance. This problem can be minimized by introducing a relationship between residual energy of nodes and round time within a cluster.

When the size of WSNs exceeds certain level, the distance between the $\mathrm{CH}$ and base station increases significantly, creating problems for single hop communications in LEACH [26]. Multi-hop LEACH (M-LEACH) solved the above issue by using multihop communication between the $\mathrm{CH}$ s and the base station. In Table 3, we compare LEACH and its various extensions based on the communication pattern considered, the assumption made about the mobility of sensor nodes, the achieved level of scalability, the used clustering approach, in addition to the achieved energy-efficiency level and the generated overhead. 
Table 3. Comparison of various extensions of LEACH protocol.

\begin{tabular}{ccccccc}
\hline Protocol & $\begin{array}{c}\text { Communication } \\
\text { Pattern }\end{array}$ & $\begin{array}{c}\text { Mobility } \\
\text { Assumption }\end{array}$ & Scalability & $\begin{array}{c}\text { Clustering } \\
\text { Approach }\end{array}$ & $\begin{array}{c}\text { Energy } \\
\text { Efficiency }\end{array}$ & Overhead \\
LEACH & Single-Hop & Stationary nodes & Low & Distributed & Moderate & High \\
A-LEACH & Chain-Based & Stationary nodes & Low & Distributed & Low \\
LEACH-EE & Multi-Hop & Stationary nodes & Very High & Distributed & High & High \\
LEACH-M & Multi-Hop & Mobile nodes & High & Distributed & Low & High \\
LEACH-F & Single-Hop & Stationary nodes & Low & Centralized & High & High \\
LEACH-DE & Single-Hope & Stationary nodes & Low & Distributed & High \\
IB-LEACH & Single Hop & Stationary nodes & Low & Distributed & High & High \\
LEACH-PRO & Single-Hop & Stationary nodes & High & Distributed & High & Moderate \\
\hline
\end{tabular}

Many LEACH related approaches continue to be proposed in the literature. A recent study presented a detailed taxonomy of such existing works [27]. Among the aspects addressed in this study are the determination of the number of clusters [28] and the consideration of the devices' mobility [29]. While these aspects are essential, they are not covered in this paper which focuses more on the routing aspects through the design of an efficient selection of cluster heads.

\section{Our Proposed Scheme}

While LEACH is an interesting routing protocol for WSNs it suffers from a number of weaknesses that affect its performance. Such weaknesses are due to a number of factors related to its operation, as highlighted below.

- Ignoring the residual energy of sensor nodes when selecting the $\mathrm{CH}$ in next rounds. Therefore, if a sensor node with low residual energy is chosen as the $\mathrm{CH}$, then it dies quickly. Hence, the robustness of the network is affected and its lifetime degrades.

- The CHs located at the boundary of the clusters would dissipate more energy and thus decrease the overall performance of the network.

- $\mathrm{CHs}$ located far away from the BS consume more energy for transmitting data compared to $\mathrm{CHs}$ closer to the BS. This can compromise the network life time.

To overcome these issues and improve the performance of $\mathrm{LEACH}$, a new variant named LEACH enhanced with probabilistic cluster head selection (LEACH-PRO) is proposed. LEACH-PRO introduces a probabilistic mechanism in which $\mathrm{CH}$ selection is made based on two metrics: The maximum residual energy and the minimum distance to BS. LEACH-PRO has similar design logic as LEACH since both protocols operate in rounds. Each round completes its operations in two phases; setup phase and steady state phase. The main difference lies in the $\mathrm{CH}$ selection process and the fact that sensors located at the border of the sensing field are excluded from participating in the $\mathrm{CH}$ selection process if they satisfy the conditions set in Equation (9). LEACH-PRO detailed operational steps are illustrated in Figure 3. We assume a static deployment of sensor nodes in the sensing field with the coordinates of each node determined a priori as $\left(x_{i}, y_{i}\right)$ to easily identify borderline nodes. Each node generates a random number and saves it in its memory. However, nodes at the borderline must be selected as $\mathrm{CM}$ only and excluded from the $\mathrm{CH}$ selection process.

It is worth noting that several other parameters could be determining for keeping a cluster active for a maximum duration. Among these parameters, there is the number of clusters in the network as well as the update frequency of the cluster head selection. These factors are discussed in several papers in the literature such as $[30,31]$. While in this paper we focused on clusters with constant load, our proposed algorithm is compatible with several strategies in the literature, such as those discussed in [32], which are based on a dynamic determination of the number of clusters. 


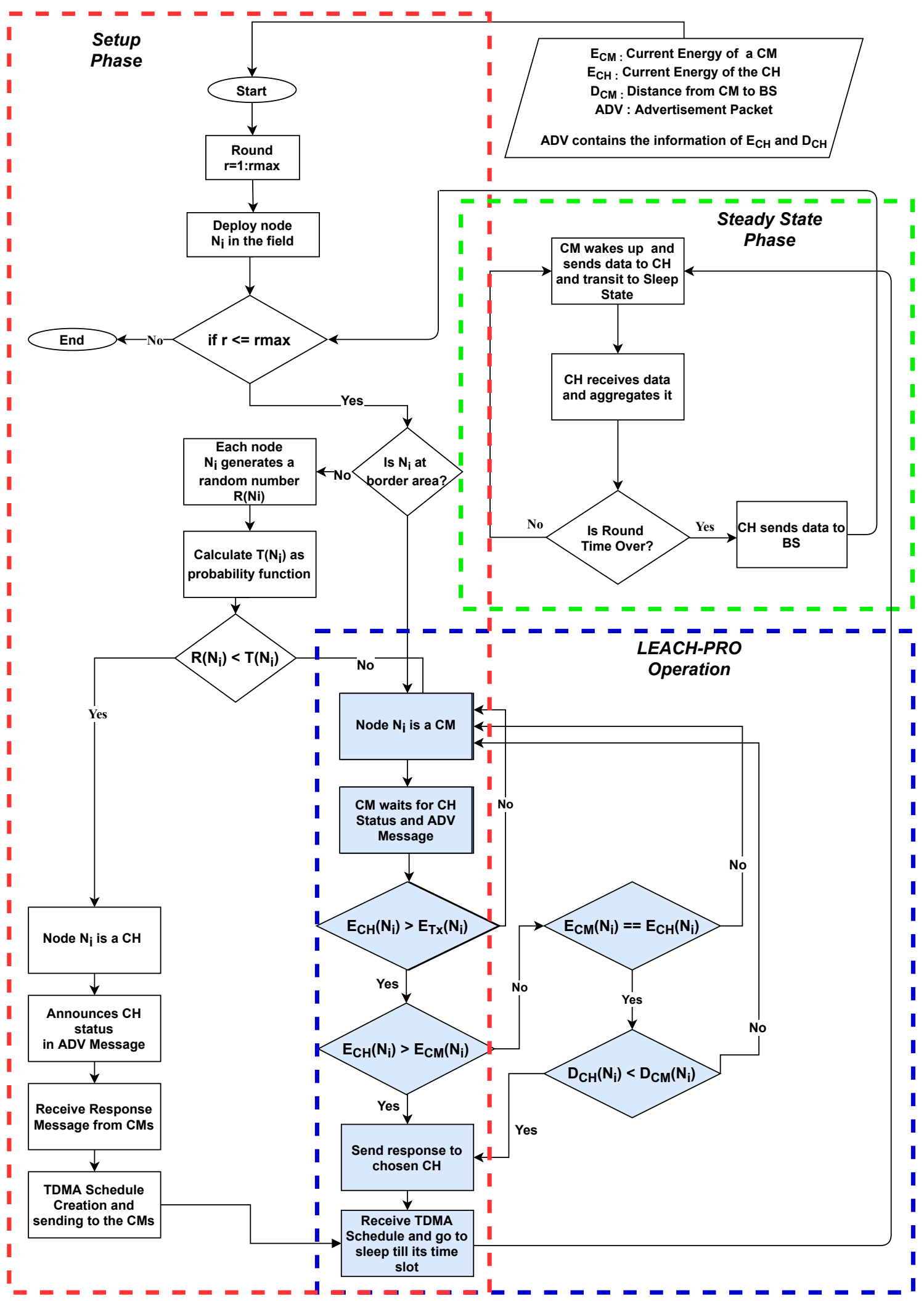

Figure 3. LEACH-PRO working principle.

\subsection{Role of a Border Sensor Node}

The node that resides in the borderline of the sensing field or closer to it is considered as a border node. The node selected as $\mathrm{CH}$ must operate and communicate with its neighbors efficiently. When clusters are formed, almost in circular fashion, each $\mathrm{CH}$ broadcasts its 
ADV message to its neighbors. Such message is transmitted to all members of each cluster. A border node can cover its communication range either side of the sensing field, therefore, if it is selected as $\mathrm{CH}$, it cannot communicate with its CMs effectively. To avoid such issue, LEACH-PRO excludes border nodes from participating in the $\mathrm{CH}$ selection process to improve the overall performance.

$$
\begin{aligned}
& x_{f-1} \geq x_{i} \geq x_{0+1} \\
& y_{f-1} \geq y_{i} \geq y_{0+1}
\end{aligned}
$$

Let us assume that the coordinates of each node $\left(x_{i}, y_{i}\right)$ are predetermined when the nodes are distributed in the sensing field. Thus, any node that satisfies the conditions set in Equation (9) is eligible to participate in the $\mathrm{CH}$ selection process; otherwise, it is excluded. These conditions are defined based on Figure 4 which illustrates the area in the sensing field where border nodes are located. Nodes in this area cannot be selected as $\mathrm{CH}$ in next rounds and are declared as CMs.



Sensor node participating in the $\mathrm{CH}$ selection process

Border sensor node excluded from the $\mathrm{CH}$ selection process

Figure 4. Border sensor nodes distribution in a sensing field.

\subsection{Advertisement Packet Message (ADV PKT)}

Generally, for clusters formation a threshold number $T\left(N_{i}\right)$ is calculated based on the probability function as given in Equation (10). If the randomly generated $R\left(N_{i}\right)$ number between 0 and 1 is less than $T\left(N_{i}\right)$ then the node $\left(N_{i}\right)$ will be selected as $\mathrm{CH}$ from the set $G$ of nodes that have not been elected as $\mathrm{CH}$ in the previous $1 / \mathrm{p}$ rounds. The first round of LEACH-PRO is similar to that of LEACH but next rounds will follow the working principle of LEACH-PRO, as illustrated in Figure 3. ADV PKT is a control packet with a size of 200 bits as set in our simulation, that contains the current energy level of the $\mathrm{CH}$ node, the distance separating it from the BS and its cluster Id denoted by $E_{C H}, D_{C H}$ and $C_{i d}$ respectively as shown in Figure 5.

$$
T\left(N_{i}\right)= \begin{cases}\frac{p \times E_{C M} \times D_{C M}}{E_{A V G} \times D_{A V G} \times\left(1-p \times\left(r \bmod \frac{1}{p}\right)\right)} & \text { if } n \in G \\ 0 & \text { Otherwise }\end{cases}
$$


where $E_{A V G}$ is the Average Energy of System, $E_{C M}$ refers to the Current Energy of $C M$, $D_{C M}$ is the Distance separating a CM from the BS, $r$ represents the Number of Rounds, $1 / p$ denotes the Number of Nodes in each Cluster, $D_{A V G}$ is the Average Distance to the BS, and $G$ represents the set of nodes that have not been selected as $\mathrm{CH}$ in $1 / \mathrm{p}$ rounds.

\begin{tabular}{|c|c|c|}
\hline$C_{\text {id }}$ & $D_{\mathrm{CH}}$ & $\mathrm{E}_{\mathrm{CH}}$ \\
\hline 100 bits & 50 bits & 50 bits \\
\hline \multicolumn{2}{|}{200 bits }
\end{tabular}

Figure 5. Advertisement message packet format.

\subsection{Cluster Head Selection Process}

The primary metric for selecting CHs is the residual energy of a node, as it is considered an optimal parameter for achieving better performance, while the distance to the BS is considered a secondary metric in the selection process of the CHs. In this regards, the following three cases are inspected. When a node is elected as a $\mathrm{CH}$ in the second round, it generates an ADV message and broadcasts it towards its CMs, as shown in Figure 6a.

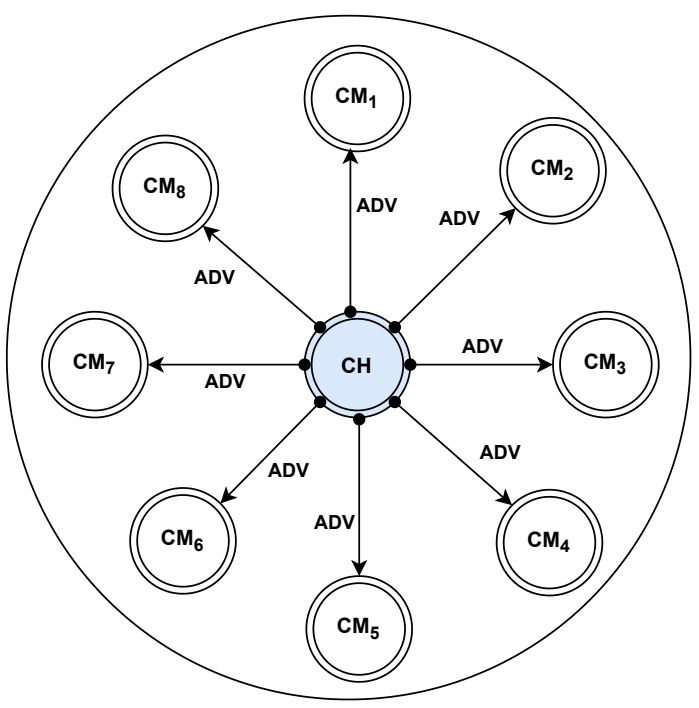

(a) Step 1: ADV to CM

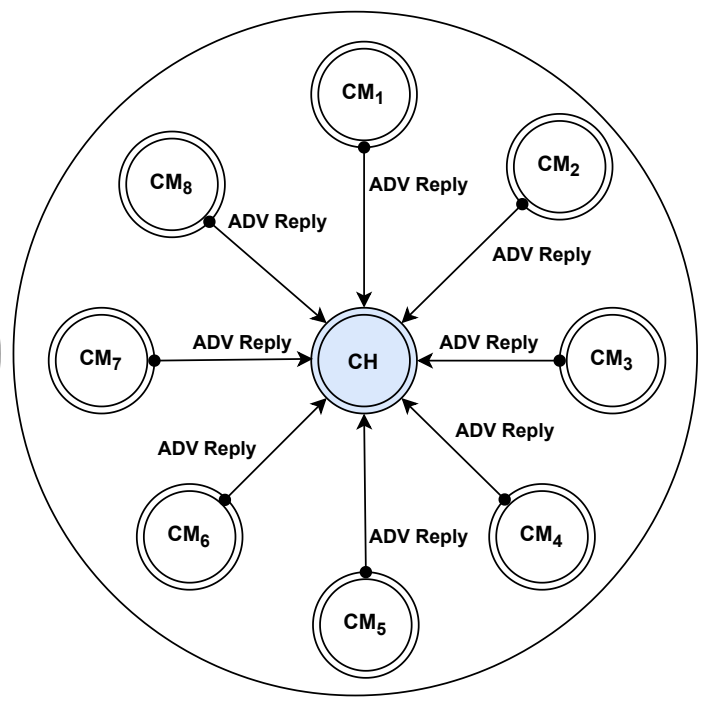

(b) Step 2: Reply to $\mathrm{CH}$

Figure 6. Selection of the energy-efficient node as CH. (a) Step 1: ADV to CM; (b) Step 2: Reply to CH.

\subsubsection{Case1: $E_{C H}>E_{C M}$}

Suppose that every $\mathrm{CM}$ in a cluster has less residual energy than the $\mathrm{CH}$. Hence, each $\mathrm{CM}$ directly responds to join that specific $\mathrm{CH}$, as shown in Figure $6 \mathrm{~b}$.

\subsubsection{Case2: $E_{C M}>E_{C H}$}

In this case, a $\mathrm{CM}$ with a higher residual energy than the $\mathrm{CH}$ generates a new ADV message and broadcasts it to its neighbors as a dominant $\mathrm{CM}$ in a cluster. Upon receiving this new ADV message each $\mathrm{CM}$ including the previously declared $\mathrm{CH}$, discards the first received ADV message based on the $C_{i d}$ information and responds to the new $\mathrm{CH}$, as illustrated in Figure 7b. 




(a) Dominant node broadcasts a new ADV message

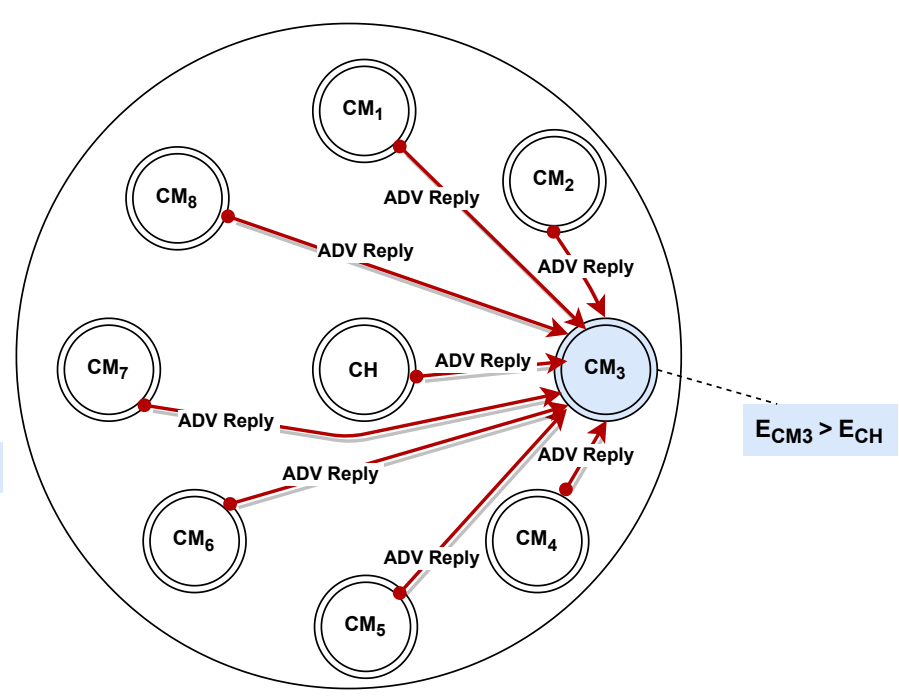

(b) CMs Reply to the dominant node

Figure 7. Maximum Residual Energy Node Broadcast ADV Message. (a) Dominant node broadcasts a new ADV message; (b) CMs Reply to the dominant node.

\subsubsection{Case3: IF $E_{C M}=E_{C H}$}

Finally, this case cannot be resolved without considering the distance separating both nodes from the BS. If a CM within a cluster has similar residual energy as that of the declared $\mathrm{CH}$, then this $\mathrm{CM}$ will check the distance to the $\mathrm{BS}$ metric. If this $\mathrm{CM}$ is closer to the $\mathrm{BS}$ than the currently declared $\mathrm{CH}$ then, the $\mathrm{CM}$ declares itself as $\mathrm{CH}$ and generates a new ADV message and broadcasts it to its neighbors in similar way as in case 2. Otherwise, the operation will be the same as in case 1 . The operation of case 3 is illustrated in Figure 8.

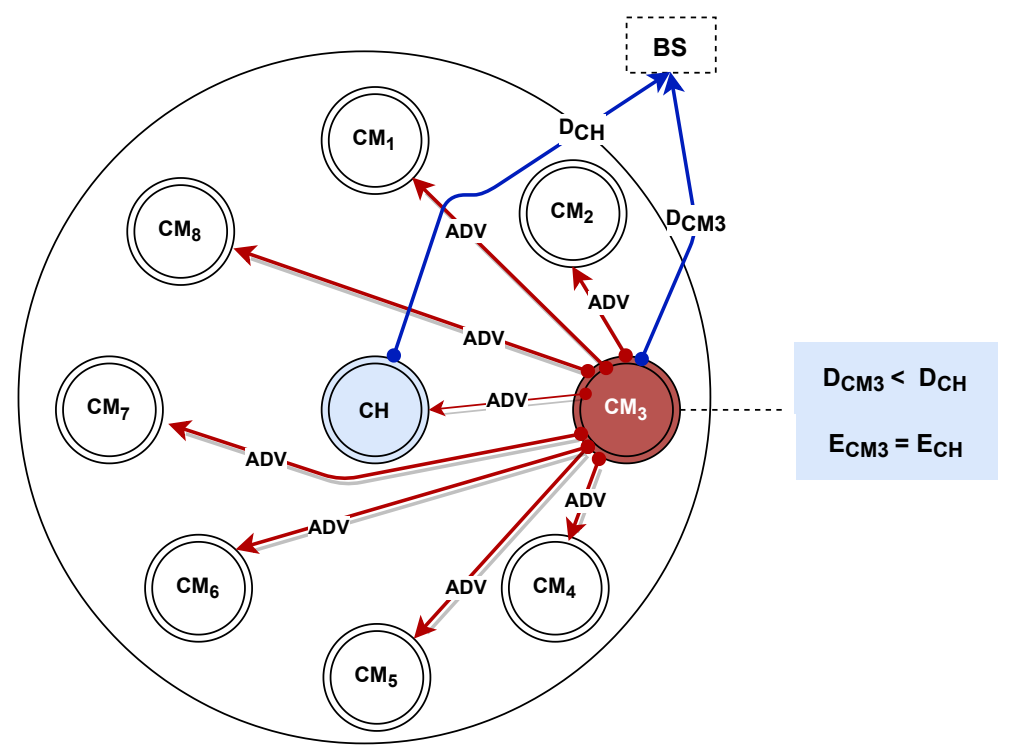

Figure 8. Optimal node declaration as $\mathrm{CH}$ with nearest distance to BS.

Finally, after selecting the optimal nodes as $\mathrm{CHs}$, each $\mathrm{CH}$ defines a TDMA schedule and sends it to its CMs. Upon reception of this TDMA frame each CM switches to sleep state until its time slot is reached. The remaining operations of LEACH-PRO are similar to that of LEACH. 


\subsection{Complexity Analysis}

The computational complexity of LEACH-PRO is very low and allows a fast convergence. The latter is of the same order of magnitude as the original LEACH protocol [33]. Indeed, there is no combinatorial here that would make the execution time important, so the execution time is of the order of $o(1)$. However, if one looks at the functional complexity, by counting the number of messages exchanged, the complexity of our protocol is reduced since the edge nodes (i.e., border sensor nodes shown in Figure 4) do not participate as $\mathrm{CHs}$. For more details about the functional complexity, one could refer to [33].

\section{Results and Discussion}

A simulation environment is created using MATLAB-2015A where DT, LEACH and LEACH-PRO are implemented to evaluate and compare their performance under different scenarios. The simulation parameters used are similar to those used in many state of the art works in this topic. The main performance evaluation metrics used are NDR (Nodes Death Rate), SRE (System Remaining Energy) and Packets to BS. NDR is calculated by measuring the First Node Death rate (FND), Half of Nodes Death rate (HND) and $90 \%$ of nodes death rate. Similarly, SRE is also calculated by measuring the remaining energy in percentage as 10\% SRE, 50\% SRE and 90\% SRE to analyze the overall performance of the protocols. Packets to BS refers to the number of packets sent towards the base station. A summary of the simulation setting is presented in Table 4.

Besides the above metrics, packet delivery ratio and end-to-end (E2E) delay measurements are very important metrics as well since they will uncover any potential negative effect of the proposed LEACH-PRO scheme. Indeed, a decrease in packet delivery ratio and/or a high end-to-end delay will make LEACH-PRO unsuitable for certain application scenarios where low delay and high reliability are required. To investigate these two metrics thoroughly we will measure them in the experiments to be conducted in the monitoring environment setup at QUEST, in Nawabshah and the results will be reported in another experimental paper.

Table 4. Summary of simulation settings.

\begin{tabular}{cc}
\hline Parameters & Values \\
\hline MATLAB Version & $2015 / \mathrm{R}$ \\
Node Deployment Area & $200 \mathrm{~m} \times 300 \mathrm{~m}$ \\
Network Sizes (Nodes Density) & $50,100,200,500$ \\
Initial Energy (J) of each node & $0.5 \mathrm{~J}$ \\
$E_{T x-e l e c}$ & $50 \mathrm{~nJ} / \mathrm{bit}$ \\
$E_{R x-e l e c}$ & $50 \mathrm{~nJ} / \mathrm{bit}$ \\
$\in_{\text {amp }}$ & $100 \mathrm{pJ} / \mathrm{bit} / \mathrm{m}^{2}$ \\
Control Packet Length & $200 \mathrm{Bits}$ \\
Data Packet Length & $4000 \mathrm{Bits}$ \\
Number of nodes in Cluster & 10 \\
Probability & 0.1 \\
Simulation Rounds & 4000 \\
Position of BS & $(100,300)$ \\
\hline
\end{tabular}

\subsection{Analysis of Network Lifetime and NDR}

The network lifetime is described as the time elapsed between initiating the network operation until the death of its last node. The scenario simulated in our experiments is similar to the topology shown in Figure 1.

In this scenario, nodes density is also varied from 50 to 500 nodes to analyze the scalability of the three protocols.

Figures 9 and 10 depict the achieved network lifetime and NDR by DT, LEACH and LEACH-PRO when applied to a WSN with sizes of 50, 100, 200 and 500 nodes respectively. The plotted results show that LEACH-PRO is outperforming the other two protocols. In DT, the source node directly transmits its data to the BS and thus consumes more 
transmit power, hence, the death rate of nodes in DT is much higher which makes the network lifetime much shorter as well. In $\mathrm{LEACH}$, instead of direct transmission the data is transmitted via CHs, thus, LEACH achieves longer lifetime than DT, but the blind CHs selection leads to a fast degradation in its performance compared to LEACH-PRO. The supremacy of this latter, in relation to network lifetime and NDR, is mainly due to its efficient probabilistic $\mathrm{CH}$ replacement based on maximum residual energy and minimum distance to BS. From Figures 9 and 10 we observe as well that the increase of the network density negatively affects the performance of all protocols, including LEACH-PRO, but this latter is still performing better than DT and LEACH.

More specifically, Figures 9a and 10a illustrate the network lifetime and NDR for a WSN of size 50. As shown in Figure 10a, the first node dies after 263 rounds, in DT, after 759 rounds, in LEACH and after 1484 rounds in LEACH-PRO. For DT, 50\% of nodes die after 502 rounds, and the entire network die after 584 rounds, whereas for LEACH 50\% of nodes consume their entire power after 1114 rounds. These results highlight that the death of the first node in LEACH-PRO occurs after the death of 1005 of nodes in DT and 50\% of nodes in LEACH. This trend continues when the network size is increased to 100 nodes. However, for a network with 200 nodes, the FND in LEACH-PRO occurs after the death of all nodes in DT but before HND occurrence in LEACH. This trend will hold with a network of 500 nodes. Such significant improvement of performance shown by LEACH-PRO is justified by the stability in finding a node with maximum residual energy to act as a $\mathrm{CH}$.

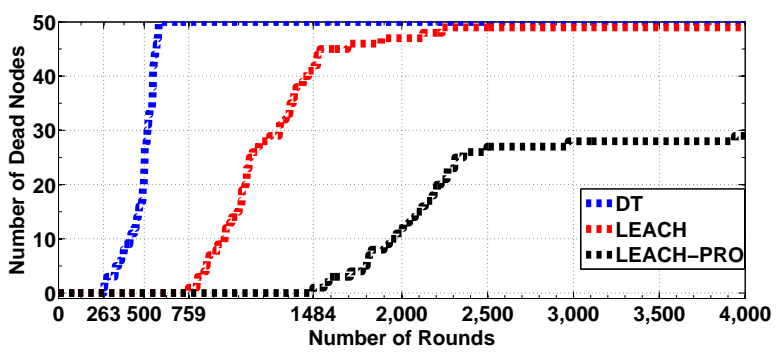

(a) Network of 50 nodes

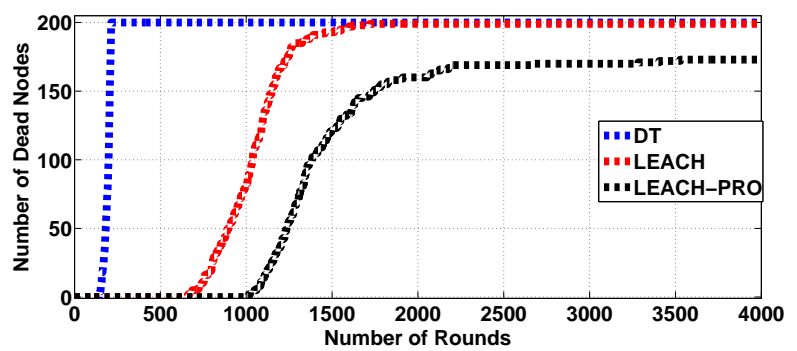

(c) Network of 200 nodes



(b) Network of 100 nodes

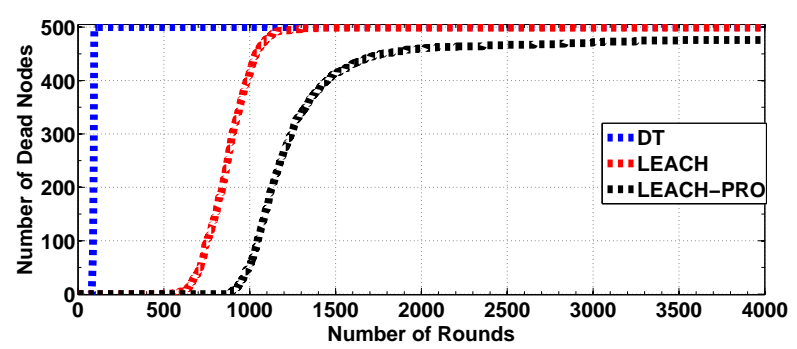

(d) Network of 500 nodes

Figure 9. The achieved lifetime for varying network densities: Number of dead nodes vs. number of rounds. Network of (a) 50, (b) 100, (c) 200, (d) 500 nodes. 


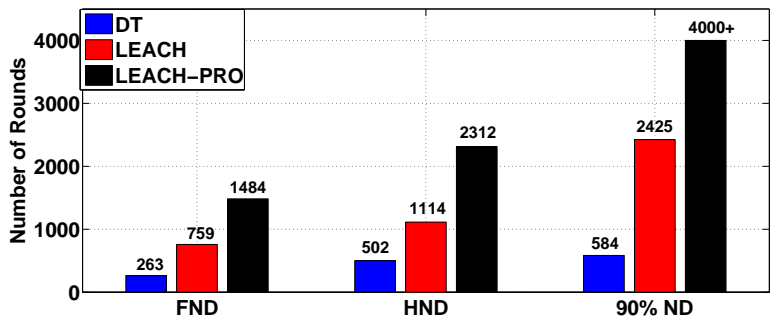

(a) Network of 50 nodes



(c) Network of 200 nodes

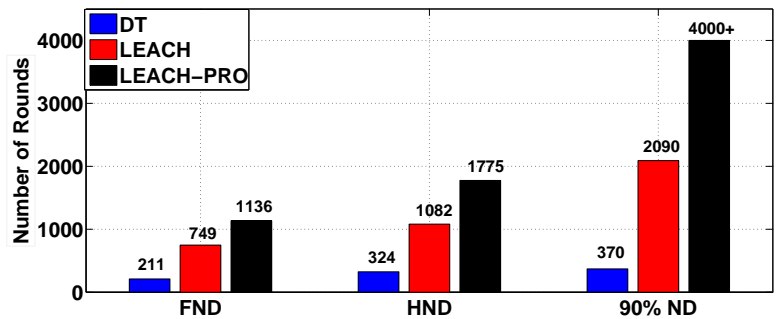

(b) Network of 100 nodes



(d) Network of 500 nodes

Figure 10. NDR evolution during the network lifetime under varying network densities. Network of (a) 50, (b) 100, (c) 200, (d) 500 nodes.

\subsection{Impact of Network Density on NDR}

Figures 11 and 12 show the evolution of FND and HND occurrence under the three protocols when the WSNs size increases. The plotted results reveal that the speed at which FND and HND occur in all protocols increases with the increase of the WSNs size but the speed increase varies. If we compare the FND occurrence between the WSNs of 50 and 500 nodes, we find that the death speed increases by a factor of 3.37, 1.36 and 1.67 in DT, LEACH and LEACH-PRO respectively. For HND, its occurrence speed has similar trend to FND where it increases by a factor of 5.52, 1.28 and 1.96 in DT, LEACH and LEACHPRO respectively. The analysis of these results tell us that although LEACH-PRO is still outperforming DT and LEACH it has a slightly higher HND speed compared to LEACH.



Figure 11. FND evolution under varying WSN densities. 




Figure 12. HND evolution under varying WSN densities.

\subsection{Analysis of Energy Consumption}

Each node in WSNs is equipped with a limited power source or battery. Therefore, the nodes energy should be utilized in an efficient manner for better sustainability of the network. The lifetime of a node is directly proportional to the speed at which its energy is being consumed. In this work, the initial energy of each node is set to 0.5 Joule.

Figures 13 and 14 represent the system residual energy (SRE) and energy consumption evaluation of DT, LEACH and LEACH-PRO for network size of 50, 100, 200 and 500 nodes respectively. As illustrated in the plotted results, LEACH-PRO is outperforming the other two protocols. DT has fast energy degradation rate as it does not use intermediate nodes for transmission towards the BS. LEACH uses its own probability function for selecting $\mathrm{CHs}$, where each node has the same probability to become a $\mathrm{CH}$, thus all nodes die quickly because of the blind $\mathrm{CH}$ selection strategy. LEACH-PRO, however, achieves the lowest degradation rate of energy due to its $\mathrm{CH}$ selection strategy that maximizes the energy efficiency of the network.

As shown in Figure 14, DT consumes more energy in less number of rounds and exhibits low performance as the size of the network increases. LEACH and LEACH-PRO both show comparable performance at the start of communication operation and do not have much difference up to 50\% of SRE. However, beyond this limit and up to $10 \%$ of SRE LEACH-PRO performs significantly better than LEACH. This supremacy of LEACH-PRO is inversely proportional to the increase of WSNs size as the performance gap between it and LEACH shrinks as the network gets larger.



(a) Network of 50 nodes

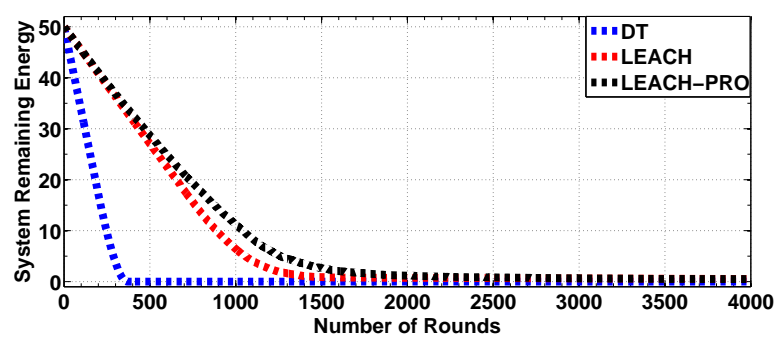

(b) Network of 100 nodes

Figure 13. Cont. 




(c) Network of 200 nodes

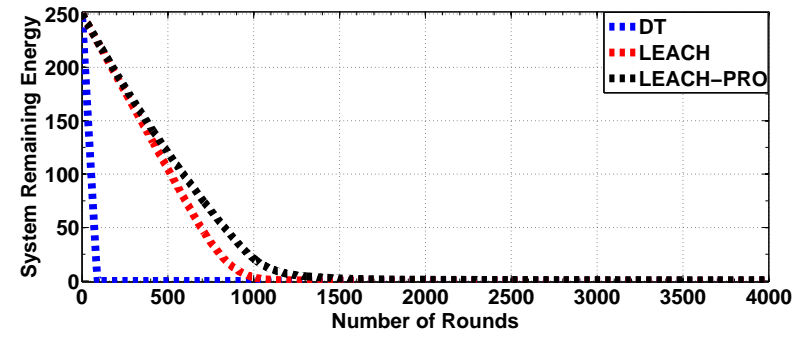

(d) Network of 500 nodes

Figure 13. System residual energy under varying network densities. Network of (a) 50, (b) 100, (c) 200, (d) 500 nodes.

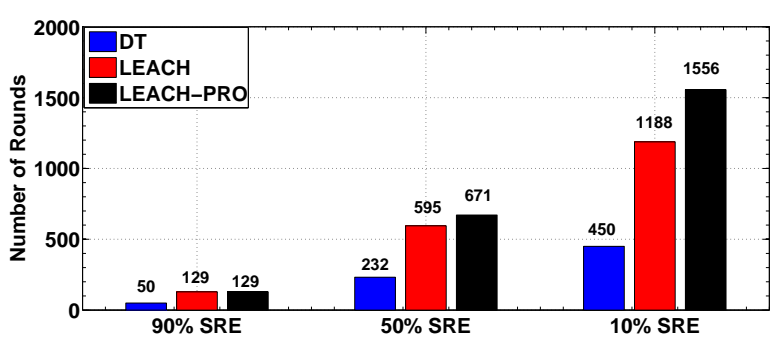

(a) Network of 50 nodes



(c) Network of 200 nodes



(b) Network of 100 nodes



(d) Network of 500 nodes

Figure 14. Energy consumption evolution under varying network densities. Network of (a) 50, (b) 100, (c) 200, (d) 500 nodes.

\subsection{Analysis of Traffic Load in the Network}

Traffic load is measured as the number of packets transmitted in the network. Higher packet transmissions directly affect the bandwidth and energy consumption, these resources are limited and thus their usage should be optimized. Therefore, it is necessary to reduce the frequent packets transmission in the network. Figure 15 illustrates the traffic load pattern generated by the three protocols. As we can see, DT generates high traffic load as compared to LEACH and LEACH-PRO due to the frequent transmissions towards the BS, and sensors die quickly after at most 500 rounds. The traffic load generated in LEACH increases gradually to stabilize after 1500 rounds, for networks of 200 and 500 nodes, and after 1000 rounds for smaller networks. LEACH-PRO has similar pattern to LEACH but the traffic load generated is almost $40 \%$ to $50 \%$ lower, especially after the first 500 rounds. 


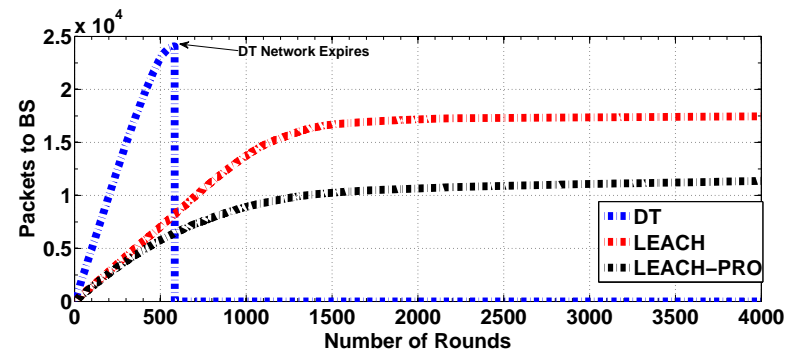

(a) Network of 50 nodes

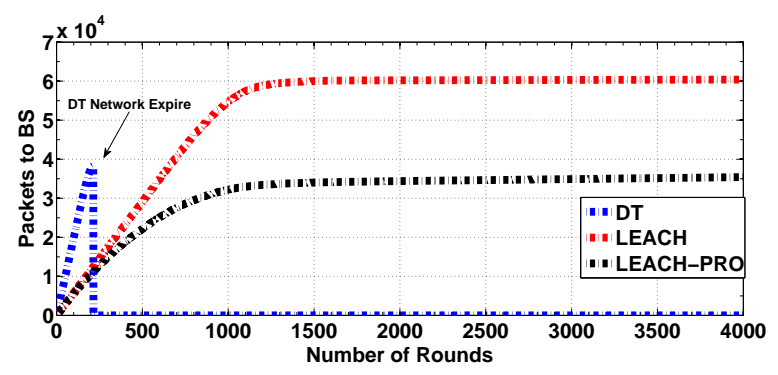

(c) Network of 200 nodes

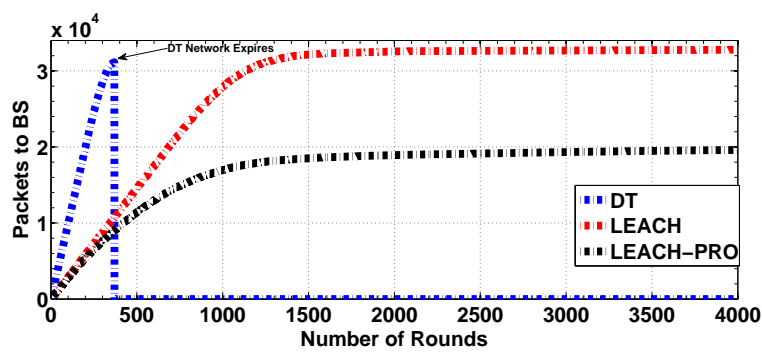

(b) Network of 100 nodes

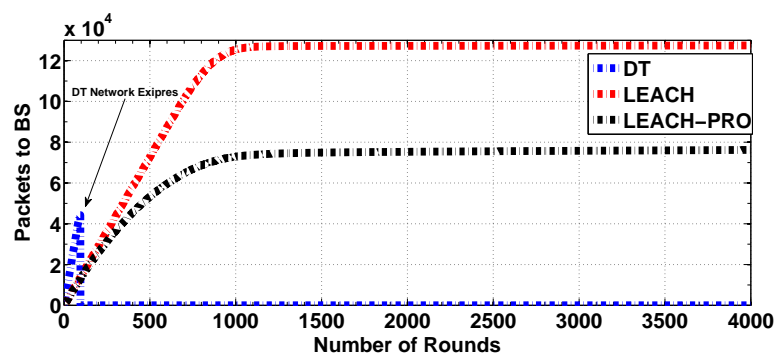

(d) Network of 500 nodes

Figure 15. Traffic load evolution under varying network densities. Network of (a) 50, (b) 100, (c) 200, (d) 500 nodes.

\section{Conclusions}

In this work, a novel energy-efficient routing protocol named LEACH-PRO was proposed to make WSNs more sustainable and optimize their operation in many smart cities use cases. This protocol was designed to overcome the limitations of LEACH by introducing the probabilistic cluster head selection based on the residual energy and the distance to the base station. Performance evaluation results highlighted that LEACHPRO outperforms both LEACH and direct transmission protocol in terms of the achieved network lifetime, node death rate speed, and residual energy depletion speed, in addition to the generated traffic load on the network. However, the results show also that as the network size increases the performance of LEACH-PRO degrades to some extent. Future works will include implementing LEACH-PRO on a hardware platform and testing its performance at the campus of QUEST in Pakistan. The promising performance shown by LEACH-PRO suggests that it can efficiently support several application scenarios in future smart cities.

Author Contributions: Conceptualization, Z.Y., I.H. and S.D.; methodology, Z.Y., I.H. and S.D.; software, Z.Y.; validation, Z.Y., I.H., S.D. and Y.H.-A.; formal analysis, S.D. and Y.H.-A.; investigation, Z.Y. and I.H.; resources, I.H.; data curation, Z.Y. and I.H.; writing—original draft preparation, Z.Y., I.H. and S.D.; writing-review and editing, S.D. and Y.H.-A.; visualization, Z.Y.; supervision, I.H. and S.D.; project administration, I.H.; funding acquisition, I.H. All authors have read and agreed to the published version of the manuscript.

Funding: This research received no external funding.

Data Availability Statement: The data presented in this study are available on request from the corresponding author. The data are not publicly available yet as it is still being used in an ongoing project.

Conflicts of Interest: The authors declare no conflict of interest. 


\section{References}

1. Khalifeh, A.; Darabkh, K.A.; Khasawneh, A.M.; Alqaisieh, I.; Salameh, M.; Alabdala, A.; Alrubaye, S.; Alassaf, A.; Al-Hajali, S.; Al-Wardat, R.; et al. Wireless Sensor Networks for Smart Cities: Network Design, Implementation and Performance Evaluation. Electronics 2021, 10, 218. [CrossRef]

2. Bensaleh, M.; Saida, R.; Kacem, Y.H.; Abid, M. Wireless Sensor Network Design Methodologies: A Survey. J. Sens. 2020, 2020, 1-13. [CrossRef]

3. Othman, M.F.; Shazali, K. Wireless Sensor Network Applications: A Study in Environment Monitoring System. Procedia Eng. 2012, 41, 1204-1210. [CrossRef]

4. Heinzelman, W.R.; Chandrakasan, A.; Balakrishnan, H. Energy-efficient communication protocol for wireless microsensor networks. In Proceedings of the 33rd Annual Hawaii International Conference on System Sciences, Maui, HI, USA, 7 January 2000; Volume 2, p. 10.

5. Ch, rakant, N.; Bijil, A.P.; Shenoy, P.D.; Venugopal, K.R.; Patnaik, L.M. Middleware service oriented rescue and crime information system (RCIS) using heterogeneous fixed nodes in WSNs. In Proceedings of the International Conference on Advanced Computing, Networking and Security, Surathkal, India, 16-18 December 2011; pp. 389-398.

6. Fanian, F.; Rafsanjani, M.K. Cluster-based routing protocols in wireless sensor networks: A survey based on methodology. J. Netw. Comput. Appl. 2019, 142, 111-142. [CrossRef]

7. Rault, T.; Bouabdallah, A.; Challal, Y. Energy efficiency in wireless sensor networks: A top-down survey. Comput. Netw. 2014, 67, 104-122. [CrossRef]

8. Toor, A.S.; Jain, A.K. Energy Aware Cluster Based Multi-hop Energy Efficient Routing Protocol using Multiple Mobile Nodes (MEACBM) in Wireless Sensor Networks. AEU Int. J. Electron. Commun. 2019, 102, 41-53. [CrossRef]

9. Kamyab, H.; Klemeš, J.J.; Fan, Y.V.; Lee, C.T. Transition to Sustainable Energy System for Smart Cities and Industries. Energy 2020, 207, 118104. [CrossRef]

10. Lim, C.; Kim, K.-J.; Maglio, P.P. Smart cities with big data: Reference models, challenges, and considerations. Cities 2018, 82, 86-99. [CrossRef]

11. Metallidou, C.K.; Psannis, K.E.; Egyptiadou, E.A. Energy Efficiency in Smart Buildings: IoT Approaches. IEEE Access 2020, 8, 63679-63699. [CrossRef]

12. Adu-Manu, K.S.; Adam, N.; Tapparello, C.; Ayatollahi, H.; Heinzelman, W. Energy-Harvesting Wireless Sensor Networks (EH-WSNs): A Review. ACM Trans. Sens. Netw. 2021, 14, 1-50. [CrossRef]

13. Tchakonte, D.T.; Simeu, E.; Tchuente, M. Lifetime optimization of wireless sensor networks with sleep mode energy consumption of sensor nodes. Wirel. Netw. 2020, 26, 91-100. [CrossRef]

14. Yetgin, H.; Cheung, K.T.K.; El-Hajjar, M.; Hanzo, L.H. A Survey of Network Lifetime Maximization Techniques in Wireless Sensor Networks. IEEE Commun. Surv. Tutor. 2017, 19, 828-854. [CrossRef]

15. Adler, R.; Buonadonna, P.; Chhabra, J.; Flanigan, M.; Krishnamurthy, L.; Kushalnagar, N.; Nachman, L.; Yarvis, M. Design and deployment of industrial sensor networks: Experiences from the north sea and a semiconductor plant. ACM SenSys 2005, 10, 1098918-1098926.

16. Geetha, V.; Kallapur, P.V.; Tellajeera, S.; Geetha, V.; Kallapur, P.V.; Tellajeera, S. Clustering in Wireless Sensor Networks: Performance Comparison of LEACH \& LEACH-C Protocols Using NS2. Procedia Technol. 2012, 4, 163-170.

17. Shi, S.; Liu, X.; Gu, X. An energy-efficiency Optimized LEACH-C for wireless sensor networks. In Proceedings of the 7th International Conference on Communications and Networking in China, Kunming, China, 8-10 August 2012; pp. 487-492.

18. Akyildiz, I.F.; Su, W.; Sankarasubramaniam, Y.; Cayirci, E. Wireless sensor networks: A survey. Comput. Netw. 2002, 38, 393-422. [CrossRef]

19. Li, X.; Xu, L.; Wang, H.; Song, J.; Yang, S.X. A differential evolution-based routing algorithm for environmental monitoring wireless sensor networks. Sensors 2010, 10, 5425-5442. [CrossRef]

20. Arumugam, G.S.; Ponnuchamy, T. EE-LEACH: Development of energy-efficient LEACH Protocol for data gathering in WSN. Eurasip J. Wirel. Commun. Netw. 2015, 2015, 1-9. [CrossRef]

21. Goyal, S.; Jain, B. Inter Cluster Balanced LEACH Algorithm for Energy Efficient Wireless Sensor Network. In Proceedings of the International Conference on Emerging Research in Computing, Information, Communication and Applications, Bangalore, India, 29-30 July 2016; pp. 535-546.

22. Kumar, S.V.; Pal, A. Assisted-leach (a-leach) energy efficient routing protocol for wireless sensor networks. Int. J. Comput. Commun. Eng. 2013, 2, 420-424. [CrossRef]

23. Li, P.; Jiang, W.; Xu, H.; Liu, W. Energy optimization algorithm of wireless sensor networks based on LEACH-B. In Proceedings of the International Conference on P2P, Parallel, Grid, Cloud and Internet Computing, Asan, Korea, 5-7 November 2016; pp. 391-404.

24. Nam, D.-H.; Min, H.-K. An energy-efficient clustering using a round-robin method in a wireless sensor network. In Proceedings of the 5th ACIS International Conference on Software Engineering Research, Management \& Applications (SERA 2007), Busan, Korea, 20-22 August 2007; pp. 54-60.

25. Azim, A.; Islam, M.M. A dynamic round-time based fixed low energy adaptive clustering hierarchy for wireless sensor networks. In Proceedings of the 2009 IEEE 9th Malaysia International Conference on Communications (MICC), Kuala Lumpur, Malaysia, 15-17 December 2009; pp. 922-926. 
26. Alsalih, W.; Hassanein, H.; Akl, S. Placement of multiple mobile data collectors in wireless sensor networks. Hoc Netw. 2010, 8 , 378-390. [CrossRef]

27. Daanoune, I.; Abdennaceur, B.; Ballouk, A. A comprehensive survey on LEACH-based clustering routing protocols in Wireless Sensor Networks. Hoc Netw. 2021, 114, 102409. [CrossRef]

28. Kirsan, A.S.; Rasyid, M.U.H.A.; Syarif, I. Efficient Energy for Cluster Head Selection using New LEACH-based routing protocol in Wireless Sensor Network. In Proceedings of the 2019 International Electronics Symposium (IES), Surabaya, Indonesia, 27-28 September 2019; pp. 70-75.

29. Thapani, P.; Arunachalam, M.; Sundarraj, D. An energy-efficient clustering and multipath routing for mobile wireless sensor network using game theory. Int. J. Commun. Syst. 2020, 33, e4336. [CrossRef]

30. Elhoseny, M.; Farouk, A.; Zhou, N.; Wang, M.; Abdalla, S.; Batle, J. Dynamic Multi-hop Clustering in a Wireless Sensor Network: Performance Improvement. Wirel. Pers. Commun. 2017, 95, 3733-3753. [CrossRef]

31. Khalifeh, A.; Abid, H.; Darabkh, K.A. Optimal Cluster Head Positioning Algorithm for Wireless Sensor Networks. Sensors 2020, 20, 3719. [CrossRef]

32. Wohwe Sambo, D.; Yenke, B.O.; Förster, A.; Dayang, P. Optimized Clustering Algorithms for Large Wireless Sensor Networks: A Review. Sensors 2019, 19, 322. [CrossRef]

33. Dzaferagic, M.; Kaminski, N.; Macaluso, I.; Marchetti, N. Relation between functional complexity, scalability and energy efficiency in WSNs. In Proceedings of the 2017 13th International Wireless Communications and Mobile Computing Conference (IWCMC), Valencia, Spain, 26-30 June 2017; pp. 675-680. [CrossRef] 